\title{
Erneute Zunahme der Fälle von Kindsmisshandlungen
}

\section{Markus Wopmann}

Dr. med., Leiter der Fachgruppe Kinderschutz der schweizerischen Kinderkliniken, Chefarzt der Klinik für Kinder und Jugendliche Kantonsspital Baden, Mitglied FMH

Im Jahre 2016 wurden im achten Jahr in Folge die Kinder erfasst, die wegen vermuteter oder sicherer Kindsmisshandlung ambulant oder stationär an einer

\section{Im Jahr 2016 wurden an Kinderkliniken zwei Kinder registriert, die in Folge einer körper- lichen Misshandlung gestorben sind.}

schweizerischen Kinderklinik behandelt worden waren. In diesem Jahr konnten die Daten von 21 von insgesamt 25 Kinderkliniken der Schweiz bearbeitet werden, was einem Rücklauf von $84 \%$ aller Kliniken entspricht. Wiederum haben alle grossen und mittelgrossen Kinderkliniken ihre Fälle gemeldet, so dass die von uns erfassten Fälle einen sehr grossen Teil der Fälle von Kindsmisshandlung darstellen, die im letzten Jahr an schweizerischen Kinderkliniken behandelt worden waren.

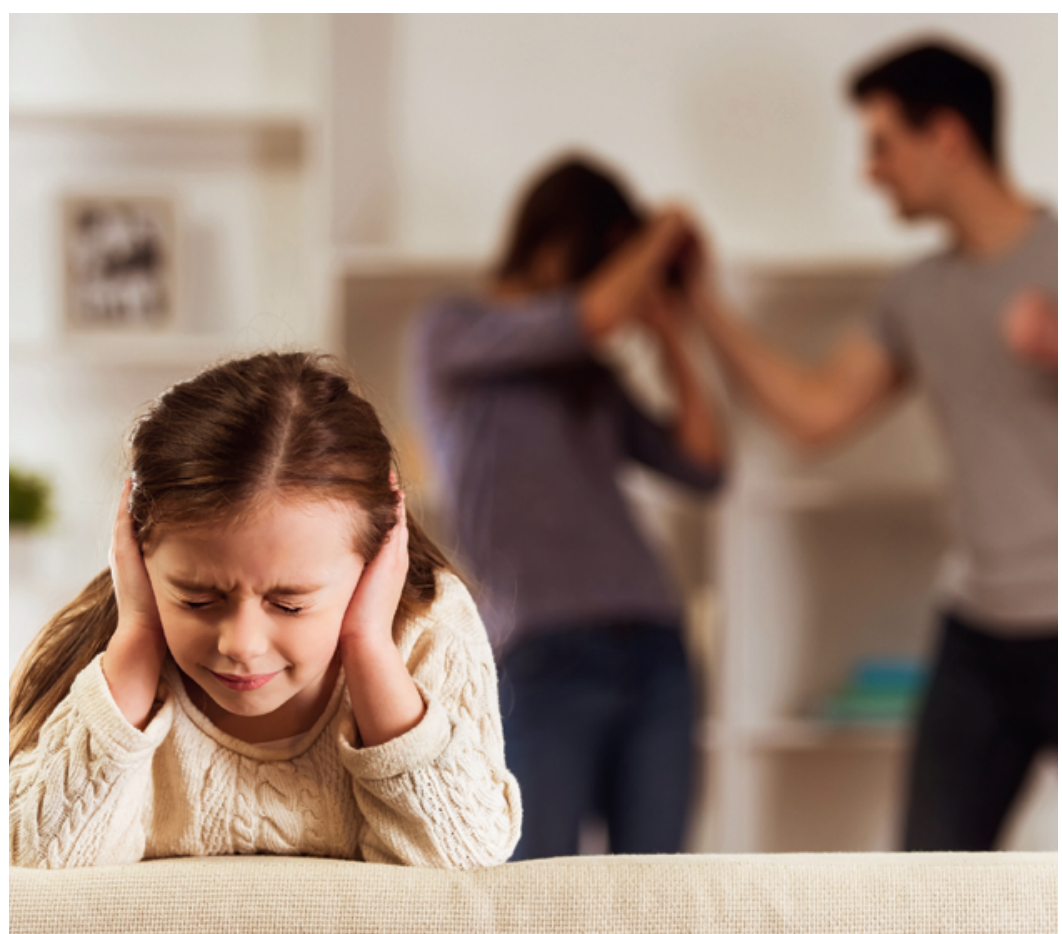

Es werden immer mehr Kinder erfasst, die wiederholt Gewalt zwischen ihren Eltern erleben.

\section{Resultate}

Von den 21 Kliniken wurden insgesamt 1575 Fälle gemeldet. Während mehrere Kliniken weniger Fälle als im Vorjahr und einige Kliniken etwa gleich viel Fälle zu verzeichnen hatten, kam es an den Universitätskliniken von Lausanne und Genf zu einer massiven $\mathrm{Zu}$ nahme von Fällen (>200 Fälle mehr!). Diese Zunahme ist in allererster Linie darauf zurückzuführen, dass diese Kliniken neu auch eingeschaltet werden, wenn es zu einem Polizeieinsatz wegen häuslicher Gewalt in Familien kommt, wo Kinder im gleichen Haushalt leben. Im Rahmen dieser Tätigkeit wird dann der psychische Zustand der Kinder beurteilt, die direkt oder indirekt von häuslicher Gewalt betroffen sind. In den verschiedenen Untergruppen ergaben sich folgende Zahlen:

\begin{tabular}{lll}
\hline Körperliche Misshandlung & 367 & $(23,3 \%)$ \\
\hline Vernachlässigung & 319 & $(20,3 \%)$ \\
\hline Psychische Misshandlung & 581 & $(36,9 \%)$ \\
\hline Sexueller Missbrauch & 306 & $(19,4 \%)$ \\
\hline Münchhausen-Stellvertreter-Syndrom & 2 & $(0,1 \%)$ \\
\hline
\end{tabular}

Gut $1 / 3$ aller Fälle betrifft Kinder, bei denen eine psychische Misshandlung diagnostiziert wurde. Dies ist darauf zurückzuführen, dass immer mehr Kinder erfasst werden, die (oft wiederholt) Gewalt zwischen den Eltern miterleben, was viele psychisch stark belastet. Mit 44\% Knaben und 56\% Mädchen ist die Geschlechterverteilung praktisch gleich wie in den letzten Jahren. Jeder 6. Misshandlungsfall betrifft ein Kind, welches jünger als ein Jahr alt ist, knapp die Hälfte aller misshandelten Kinder ist jünger als sechs Jahre.

\begin{tabular}{lll}
\hline $\begin{array}{l}\text { Geschlecht der Kinder in den } \\
\text { einzelnen Diagnosegruppen }\end{array}$ & Knaben & Mädchen \\
\hline Körperliche Misshandlung & $53,4 \%$ & $46,6 \%$ \\
\hline Vernachlässigung & $49,5 \%$ & $50,5 \%$ \\
\hline Psychische Misshandlung & $47,5 \%$ & $52,5 \%$ \\
\hline Sexueller Missbrauch & $19,9 \%$ & $80,1 \%$ \\
\hline
\end{tabular}

Es zeigt sich eine ziemlich ausgeglichene Geschlechterverteilung in allen Misshandlungsformen mit Aus- 
nahme des sexuellen Missbrauchs, wo Mädchen viermal häufiger als Knaben betroffen waren.

\begin{tabular}{lll}
\hline Sicherheit der Diagnose & & \\
\hline Sicher & 959 & $(60,9 \%)$ \\
\hline Wahrscheinlich & 242 & $(15,4 \%)$ \\
\hline Unklar & 273 & $(17,3 \%)$ \\
\hline Keine Angabe & 101 & $(6,4 \%)$ \\
\hline
\end{tabular}

Erstaunlicherweise wurde bei der psychischen Misshandlung in $84,2 \%$ die Diagnose als sicher eingestuft, bei der körperlichen Misshandlung und bei der Vernachlässigung war diese Sicherheit nur in rund der

\section{Jeder 6. Misshandlungsfall betrifft ein Kind,} welches jünger als ein Jahr alt ist, knapp die Hälfte aller misshandelten Kinder ist jünger als sechs Jahre.

Hälfte der Fälle gegeben. Sexueller Missbrauch wurde nur in 37,6\% der Fälle als sicher erachtet, dies widerspiegelt auch die Schwierigkeit dieser Diagnosestellung.

\begin{tabular}{lll}
\hline \multicolumn{3}{l}{ Täterin/Täter: Beziehung zum Kind } \\
\hline Familie & 1272 & $(80,8 \%)$ \\
\hline Bekannte/r des Kindes & 186 & $(11,8 \%)$ \\
\hline Fremdtäter & 37 & $(2,3 \%)$ \\
\hline Unbekannter Täter & 80 & $(5,1 \%)$
\end{tabular}

Über 95\% der Fälle von psychischer Misshandlung oder von Vernachlässigung finden im Familienrahmen statt, körperliche Misshandlung in $76 \%$, sexueller Missbrauch in 39,2\%. Gut 20\% der sexuellen Übergriffe werden durch Fremdtäter oder unbekannte Täter begangen.

\begin{tabular}{lcc}
\hline Täterin/Täter: Geschlecht & & \\
\hline Männlich & 717 & $(45,5 \%)$ \\
\hline Weiblich & 384 & $(24,4 \%)$ \\
\hline $\begin{array}{l}\text { Männlich und weiblich } \\
\text { (meist Eltern gemeinsam) }\end{array}$ & 351 & $(22,3 \%)$ \\
\hline Unbekannt & 123 & $(7,8 \%)$ \\
\hline
\end{tabular}

Es zeigt sich eine praktisch genau gleiche Verteilung wie im letzten Jahr. Wie zu erwarten, ist im Bereiche des sexuellen Missbrauchs der Anteil der männlichen Täter mit 87,3\% am höchsten. Auch bei der körperlichen Misshandlung und bei der psychischen Misshandlung stellen die Männer mit 41,4\% respektive 44,8\% die Hauptgruppe dar, nur bei der Vernachlässi- gung ist der Frauenanteil bei den Tätern deutlich höher als der der Männer.

\begin{tabular}{lll}
\hline Täterin/Täter: Alter & & \\
\hline Älter als 18 Jahre & 1343 & $(85,3 \%)$ \\
\hline $\begin{array}{l}\text { Jünger als 18 Jahre } \\
\begin{array}{l}\text { Jünger und älter als 18 Jahre } \\
\text { (mehrere Täter) }\end{array}\end{array}$ & 147 & $(9,3 \%)$ \\
\hline $\begin{array}{l}\text { Unbekanntes Alter / keine Angabe } \\
76\end{array}$ & $(0,6 \%)$ \\
\hline
\end{tabular}

Die jugendlichen Täter sind für 27,5\% aller Fälle von sexuellem Missbrauch verantwortlich, bei Fällen von körperlicher Misshandlung für 10\%. In den übrigen Misshandlungsformen ist ihr Anteil sehr gering.

\begin{tabular}{|c|c|c|}
\hline \multicolumn{3}{|c|}{ Meldung an die Kindes- und Erwachsenenschutzbehörde } \\
\hline $\begin{array}{l}\text { Durch eine andere Stelle bereits } \\
\text { vorgenommen }\end{array}$ & 268 & $(17,0 \%)$ \\
\hline $\begin{array}{l}\text { Durch die Kinderschutzgruppe } \\
\text { gemacht }\end{array}$ & 419 & $(26,6 \%)$ \\
\hline $\begin{array}{l}\text { Durch die Kinderschutzgruppe } \\
\text { empfohlen }\end{array}$ & 119 & $(7,6 \%)$ \\
\hline \multicolumn{3}{|c|}{ Meldung an die Strafverfolgungsbehörde } \\
\hline $\begin{array}{l}\text { Durch eine andere Stelle bereits } \\
\text { vorgenommen }\end{array}$ & 214 & $(13,6 \%)$ \\
\hline $\begin{array}{l}\text { Durch die Kinderschutzgruppe } \\
\text { gemacht }\end{array}$ & 79 & $(5,0 \%)$ \\
\hline $\begin{array}{l}\text { Durch die Kinderschutzgruppe } \\
\text { empfohlen }\end{array}$ & 72 & $(4,6 \%)$ \\
\hline
\end{tabular}

\section{Fazit}

- Weitere Zunahme der an schweizerischen Kinderkliniken registrierten Fälle von Kindsmisshandlung, was die traurige Wichtigkeit dieser Diagnose bestätigt.

- Psychische Misshandlung, vor allem durch das Miterleben von Gewalt unter den Eltern, ist ein Phänomen, das immer häufiger beobachtet wird. Leider werden auch in vielen Familien zwei oder mehr Polizeieinsätze innert eines Jahres verzeichnet, was die Bedeutung dieser Thematik noch unterstreicht.

- Im Jahr 2016 wurden an Kinderkliniken zwei Kinder registriert, die in Folge einer körperlichen Misshandlung gestorben sind. Eines dieser Kinder war jünger als ein Jahr alt, das zweite Kind zwischen eins und zwei Jahren. Dies entspricht der internationalen Erfahrung, dass das Risiko für eine schwere oder sogar tödliche Misshandlung bei ganz jungen Kindern am grössten ist.

Bildnachweis

(c) Vadimgozhda | Dreamstime.com 